\title{
Multiscale edge detection using first-order derivative of anisotropic Gaussian kernels
}

\author{
Gang Wang · Carlos Lopez-Molina · Bernard De \\ Baets
}

Received: date / Accepted: date

\begin{abstract}
Spatially scaled edges are ubiquitous in natural images. To better detect edges with heterogeneous widths, in this paper, we propose a multiscale edge detection method based on first-order derivative of anisotropic Gaussian kernels. These kernels are normalized in scale-space, yielding a maximum response at the scale of the observed edge, and accordingly, the edge scale can be identified. Subsequently, the maximum response and the identified edge scale are used to compute the edge strength. Furthermore, we propose an adaptive anisotropy factor of which the value decreases as the kernel scale increases. This factor improves the noise-robustness of small-scale kernels while alleviating the anisotropy stretch effect that occurs in conventional anisotropic methods. Finally, we evaluate our method on widely used datasets. Experimental results validate the benefits of our method over the competing methods.
\end{abstract}

Keywords multiscale edge detection · edge strength · first-order derivative of anisotropic Gaussian kernels $\cdot$ scale-space $\cdot$ noise-robustness

\section{Introduction}

Edges are fundamental visual features bridging the gap between image pixels and many computer vision tasks [16], like superpixel segmentation [44] and object detection [51]. In

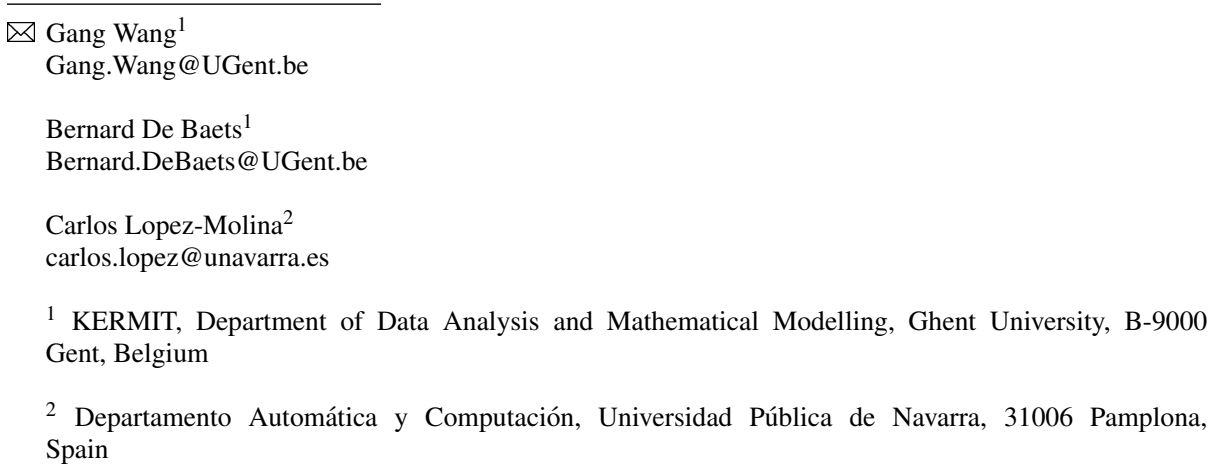


general, a good edge detector is expected to reliably detect the locations that reveal critical structural information of the image contents [6], which facilitates subsequent practical applications. Over the past several decades, numerous edge detectors have been developed. Most of them focus on two aspects: (1) computing the edge strength, and (2) binarizing the edge strength into the binary edge map. In this paper, we intend to address the former one. The edge strength (a.k.a. edge saliency [18], edginess [15] or edge response [3]) is a measure of the local intensity variation and is widely considered the most relevant representation of edges [24]. For the sake of efficiency and interpretability, the edge strength is usually obtained by differentiation-based methods. These methods can be subdivided into two categories: monoscale methods and multiscale methods.

Early monoscale methods, such as the Roberts cross method [34], the Sobel method [40] and the Prewitt method [32], employ very simple differential kernels to compute the gradients, and accordingly compute the edge strength by the magnitude of the gradients. These kernels are computationally cheap, yet are of fixed size and sensitive to noise [50]. To overcome these shortcomings, some monoscale approaches employ kernels of which the size is alterable. Marr and Hildreth [26] use the Laplacian of Gaussian as convolution kernel. By adjusting the kernel size through the scale of the Gaussian function, the Laplacian of Gaussian kernels are applicable to either thin edges or wide edges. Nevertheless, it is the Canny detector [5] that has gained the most prominent position in the literature. For detecting isolated step edges corrupted by additive white Gaussian noise, Canny derived the theoretically optimal operator based on three criteria, i.e., good detection, good localization and low spurious response. To make the detector computationally efficient, he uses the firstorder derivative of isotropic Gaussian (FDIG) kernel as an approximation of the optimal operator. However, the scale choice in the Canny method is a dilemma. Although obtaining a better localization, a smaller scale is more sensitive to noise compared with a coarser scale. It is worth noting that Canny also agrees with the need for kernels with multiple scales and multiple orientations [5]. Employing the Canny method as a standard, many edge detectors have been developed to revise the Canny method [7,13,48]. Zhang et al. [50] proposed a method using a directional version of the first-order derivative of anisotropic Gaussian (FDAG) kernel, ensuring a good noise-robustness while maintaining a good detection for adjacent edges. The anisotropy of this method makes an indispensable contribution to the improvement of the signal-to-noise ratio (SNR) [50]. However, due to the fact that the blurring extents of an FDAG kernel along two orthogonal directions are different, the use of the FDAG kernel can incur an anisotropy stretch effect. As a consequence, in the resulting edge strength map, there are significant but factitious responses in the vicinity of blobs and corners, and subsequently, these factitious responses will lead to spurious results when a low threshold is used to detect weak edges [50].

The monoscale edge detectors mentioned above are mostly designed to detect step edges, but edges usually extend over different widths [31]. Due to the focal properties of sensors, the penumbral phenomenon of light, etc., in natural images, one can generally find spatially scaled edges of which the intensity discontinuities vary over different widths [9]. Therefore, the assumptions of most monoscale edge detectors do not conform to actual situations, and as such, those monoscale detectors are weak at detecting edges with heterogeneous widths $[41,9]$.

Multiscale edge detection, which is inspired by the mechanism of the human visual system [2], has gained increasing attention [24]. Following an early proposal [36], authors have developed different strategies to implement the multiscale detection. One popular strategy is to fuse multiple edge strength maps that are obtained at different scales. Bao et al. [3] proposed such a method based on scale multiplication. Exploiting the fact that the product 
is large only when all of the factors are large [35], this method computes the product of the gradients obtained at two different scales as the edge strength. Adopting a similar strategy, Shui and Zhang [38] developed a method based on both isotropic and anisotropic Gaussian kernels. This method obtains the edge strength by computing the geometric mean of two gradient maps. One gradient map is obtained by isotropic kernels, while the other one is obtained by anisotropic kernels. In this way, the anisotropy stretch effect can also be mitigated. Nonetheless, the employed isotropic kernels are sensitive to noise [38], which would lead to factitious responses in the vicinity of true edges [50]. Moreover, both [3] and [38] only consider gradient maps at two user-specified scales, and therefore still have limitations in detecting edges with heterogeneous widths.

There are also multiscale methods that find the optimal scale for each edge. Elaborating the need for a multiscale framework, Elder et al. [9] proposed a scale estimation method based on the second-order derivative of the image intensity, but prior knowledge of sensor noise is required. Building on the scale-space theory, Lindeberg [18] developed an automatic scale selection method using the normalized and directional isotropic Gaussian kernels. A family of kernels covering all possible scales and orientations is employed to filter the image, and for each pixel, the maximum response among all the kernels is retained to form the edge strength map. We refer to this scheme as maximum aggregation. Note that Lindeberg's method is rotationally invariant because a set of kernels with all possible orientations is employed. However, as Lindeberg stated, there is a systematic bias in the edge strength map produced by his method [18]. For a spatially scaled edge, the bias renders the obtained edge strength smaller than the true edge strength. Moreover, like other methods based on isotropic kernels, Lindeberg's method is not robust to noise.

In this paper, we analyze the advantages of anisotropic kernels and multiscale detection over isotropic kernels and monoscale detection, respectively. Exploiting these advantages, we present an edge detection method based on the Multiscale version of the First-order Derivative of Anisotropic Gaussian (MFDAG) kernel. It has been argued that the conventional anisotropic Gaussian kernels $[38,50]$ for edge detection cannot be applied in scalespace directly. To address this problem, inspired by Lindeberg's strategy [18], we propose a normalization method that enables the MFDAG kernels to yield a maximum response at the scale of the edge to be detected. Accordingly, the edge scale can be identified. It is also observed that compared with the true edge strength, the maximum response has a bias that is related to the edge scale. To reduce this bias, the maximum response and the identified edge scale are subsequently used to compute the edge strength. In addition, to address the anisotropy stretch effect brought by the conventional anisotropic Gaussian kernels, we propose an adaptive anisotropy factor of which the value decreases as the kernel scale increases. In this way, the adaptive anisotropy factor improves the noise-robustness of small-scale kernels while alleviating the anisotropy stretch effect that occurs in conventional anisotropic methods $[38,50]$. Eventually, by incorporating a binarization procedure, a full-fledged framework for multiscale edge detection is developed.

This paper is organized as follows. Section 2 elaborates some preliminary concepts that are of interest to this work. In Section 3, we present the normalized FDAG kernels in scalespace. Based on a noise-robustness analysis in terms of the SNR, we propose an adaptive anisotropy factor in Section 4. Section 5 presents the proposed multiscale framework for edge detection. Experimental results as well as discussions are included in Section 6, while Section 7 lists our conclusions. 


\section{Related work}

This section presents several concepts related to this work: the scale-space representation of images, the directional version of the FDIG kernel and the directional version of the FDAG kernel.

2.1 The scale-space representation and its derivative

In the computer vision field, the scale-space framework has been applied to a large variety of topics, such as image matching [20], line detection [46] and blob reconstruction [45]. The scale-space representation of a signal is obtained by convolving the signal with Gaussian kernels [19]. Considering the fact that the magnitude of a non-normalized Gaussian kernel decreases over scales, Lindeberg introduced a $\gamma$-parameterized normalized Gaussian kernel as follows [18]:

$$
\hat{g}(\mathbf{x} ; \sigma)=\sigma^{2 \gamma} \cdot g(\mathbf{x} ; \sigma),
$$

where $\mathbf{x}=[x, y]^{T}$ denotes the planar coordinates, $\sigma \in \mathbb{R}_{+}$stands for a scale in scale-space, $\gamma \in$ $\mathbb{R}_{+}$is referred to as the scale normalization factor and $g(\mathbf{x} ; \sigma)$ represents the non-normalized isotropic Gaussian kernel:

$$
g(\mathbf{x} ; \sigma)=\frac{1}{2 \pi \sigma^{2}} \exp \left(-\frac{\mathbf{x}^{T} \mathbf{x}}{2 \sigma^{2}}\right) .
$$

From Eq. (1), it is easy to get the $\gamma$-parameterized normalized version of the FDIG kernel:

$$
\hat{g}^{\prime}(\mathbf{x} ; \sigma)=\sigma^{2 \gamma} \cdot g^{\prime}(\mathbf{x} ; \sigma),
$$

where $g^{\prime}(\mathbf{x} ; \sigma)$ is the first-order derivative of $g(\mathbf{x} ; \sigma)$ w.r.t. the argument $x$ in $\mathbf{x}$ :

$$
g^{\prime}(\mathbf{x} ; \sigma)=-\frac{x}{\sigma^{2}} \cdot g(\mathbf{x} ; \sigma)
$$

According to Lindeberg's scale-space framework [18], given a two-dimensional signal $f(\mathbf{x})$, its normalized scale-space representation $r(\mathbf{x} ; \sigma)$ is computed by filtering the signal with the $\gamma$-parameterized normalized Gaussian kernel:

$$
\begin{aligned}
r(\mathbf{x} ; \sigma) & =f(\mathbf{x}) * \hat{g}(\mathbf{x} ; \sigma) \\
& =f(\mathbf{x}) *\left(\sigma^{2 \gamma} \cdot g(\mathbf{x} ; \sigma)\right),
\end{aligned}
$$

where $*$ denotes the convolution operation. Likewise, using Eq. (3), the normalized firstorder derivative of the scale-space representation is given by:

$$
\begin{aligned}
r^{\prime}(\mathbf{x} ; \sigma) & =\left|f(\mathbf{x}) * \hat{g}^{\prime}(\mathbf{x} ; \sigma)\right| \\
& =\left|f(\mathbf{x}) *\left(\sigma^{2 \gamma} \cdot g^{\prime}(\mathbf{x} ; \sigma)\right)\right| .
\end{aligned}
$$

Lindeberg's method [18] computes the edge strength at all the scales using Eq. (6). Subsequently, for each edge, the matched scale is identified as the scale that makes the edge strength locally maximal in scale-space. 
2.2 The directional Canny kernel

As mentioned in Section 1, Canny proposed three edge detection criteria and expressed them mathematically. Accordingly, he derives an optimal operator for detecting step edges and uses a normalized FDIG kernel as an approximation. The normalized FDIG kernel in the Canny method (Canny kernel) is formulated as [5]:

$$
g_{\mathrm{C}}^{\prime}(\mathbf{x} ; \sigma)=-\frac{x}{\sigma^{2}} \cdot g_{\mathrm{C}}(\mathbf{x} ; \sigma),
$$

where $g_{\mathrm{C}}(\mathbf{x} ; \sigma)$ is the Canny-normalized isotropic Gaussian kernel, which is given by:

$$
g_{\mathrm{C}}(\mathbf{x} ; \sigma)=2 \pi \sigma^{2} \cdot g(\mathbf{x} ; \sigma) .
$$

As Canny stated, the kernel should ideally be oriented normal to the direction of the edge to be detected [5], but for the sake of computational efficiency, only the vertical and horizontal gradients are calculated to form the gradient vector. Subsequently, the magnitude (i.e., the Euclidean norm) of the gradient vector is computed as the edge strength. In this paper, to explore the ideal performance of the Canny kernel, we study the case that the Canny kernel is exactly oriented normal to the direction of the edge. We rotate the Canny kernel in Eq. (7) with an orientation $\theta$, thereby getting its directional version:

$$
g_{\mathrm{C}}^{\prime}(\mathbf{x} ; \sigma, \theta)=-\frac{[\cos \theta, \sin \theta] \mathbf{x}}{\sigma^{2}} \exp \left(-\frac{1}{2 \sigma^{2}} \mathbf{x}^{T} \mathbf{R}_{\theta}^{T} \mathbf{R}_{\theta} \mathbf{x}\right),
$$

where

$$
\mathbf{R}_{\theta}=\left[\begin{array}{cc}
\cos \theta & \sin \theta \\
-\sin \theta & \cos \theta
\end{array}\right]
$$

denotes the rotation matrix and $\theta \in[0,2 \pi$ [ stands for the orientation. Since the absolute values of the responses at $\theta$ and $\theta+\pi$ are identical, it is equivalent to set the orientation interval as $\theta \in[0, \pi[$.

Since the direction of the observed edge is unknown beforehand, a family of Canny kernels covering all possible orientations should be used to filter the image signal. The maximum response among all the orientations is then selected as the edge strength.

Over the past two decades, the Canny method has gained popularity in edge detection. Nevertheless, it should not be taken as the final solution to edge detection [4]. Even Canny himself also referred to his proposal as non-final and envisaged that kernels with different widths (i.e., scales) and orientations shall be used to cope with the edges in real images [5].

2.3 The first-order derivative of an anisotropic Gaussian kernel

A key development in adapting the Canny kernel to multiple edge topologies is the use of anisotropic Gaussian kernels [38,50,37]. Anisotropic Gaussian kernels have also found applications in corner detection [39] and ridge detection [25].

The directional version of an anisotropic Gaussian kernel is defined by [43]:

$$
g(\mathbf{x} ; \sigma, \varphi, \theta)=\frac{1}{2 \pi \varphi \sigma^{2}} \exp \left(-\frac{1}{2 \sigma^{2}} \mathbf{x}^{T} \mathbf{R}_{\theta}^{T}\left[\begin{array}{cc}
1 & 0 \\
0 & \varphi^{-2}
\end{array}\right] \mathbf{R}_{\theta} \mathbf{x}\right)
$$


where $\varphi \geq 1$ denotes the anisotropy factor. Note that the anisotropic Gaussian kernel in Eq. (11) reduces to an isotropic version when $\varphi=1$. From Eq. (11), the FDAG kernel is given by:

$$
g^{\prime}(\mathbf{x} ; \sigma, \varphi, \theta)=-\frac{[\cos \theta, \sin \theta] \mathbf{x}}{\sigma^{2}} g(\mathbf{x} ; \sigma, \varphi, \theta) .
$$

In FDAG-based methods [38, 50], for a specified scale, a family of FDAG kernels covering all possible orientations is required to filter the image signal, and the maximum response is selected as the edge strength. Correspondingly, the direction of each edge is indicated by the kernel that produces the maximum response. It has been verified that the use of an anisotropy factor helps to improve the robustness to noise $[38,50]$. Nonetheless, existing FDAG-based methods only address the edge detection at one or two scales, and as such, they still have limitations in detecting edges with heterogeneous widths.

\section{The MFDAG kernels}

In this section, we propose a normalization method that enables the FDAG kernels to identify the edge scales while yielding the multiscale edge strength maps. These normalized FDAG kernels form the MFDAG kernels in scale-space.

\subsection{Modelling the scaled edges}

Many existing edge detectors are designed with the assumption that the local variation of image intensity shows a step appearance. In fact, besides step edges, there are also spatially scaled edges in real images [29]. In this paper, we model the variation of image intensity using scaled edges, which can be regarded as the convolutional result of a step edge and a Gaussian kernel:

$$
f\left(\mathbf{x} ; \omega_{0}, \theta_{0}\right)=\left(c_{0} H\left(\left[\cos \theta_{0}, \sin \theta_{0}\right] \mathbf{x}\right)+b_{0}\right) * g\left(\mathbf{x} ; \omega_{0}\right),
$$

where

$$
H(u)=\left\{\begin{array}{l}
0, \text { if } u<0 \\
1, \text { if } u \geq 0
\end{array}\right.
$$

is the Heaviside step function, and

$$
g\left(\mathbf{x} ; \omega_{0}\right)=\frac{1}{2 \pi \omega_{0}^{2}} \exp \left(-\frac{\mathbf{x}^{T} \mathbf{x}}{2 \omega_{0}^{2}}\right)
$$

is a Gaussian function, in which $\theta_{0} \in\left[0, \pi\right.$ [ denotes the normal direction of the edge, $\omega_{0} \in$ $\mathbb{R}_{+}$stands for the edge scale which determines the spatial width of intensity variation, $c_{0} \in$ $[0,1]$ is a constant that controls the true edge strength (i.e., extent of intensity change) and $b_{0}\left(b_{0}+c_{0} \leq 1\right)$ denotes the base level reflecting the background intensity. Note that a scaled edge modelled by Eq. (13) reduces to a step edge in case $\omega_{0} \approx 0$. As a matter of fact, with respect to digital images, we always have $\omega_{0}>0$, since digital images are discrete signals. In addition, practical edge detectors usually impose an intrinsic smoothing on the image to get rid of the interference from noise, and as such, we have $\omega_{0} \geq 1$ in most actual situations.

As an illustration, Fig. 1 displays several edges with different widths, and Fig. 2 shows their horizontal profiles through the image centers. 


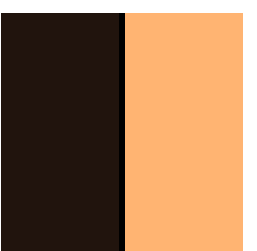

(a)

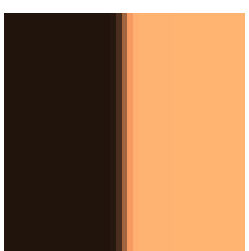

(b)

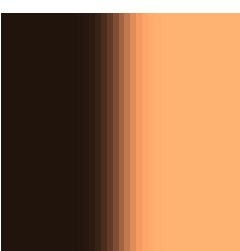

(c)

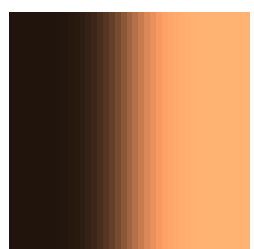

(d)

Fig. 1: Examples of edges with different widths. Their scales are $\omega_{0} \approx 0$ (a), $\omega_{0}=1$ (b), $\omega_{0}=3(\mathrm{c})$ and $\omega_{0}=5(\mathrm{~d})$, respectively.

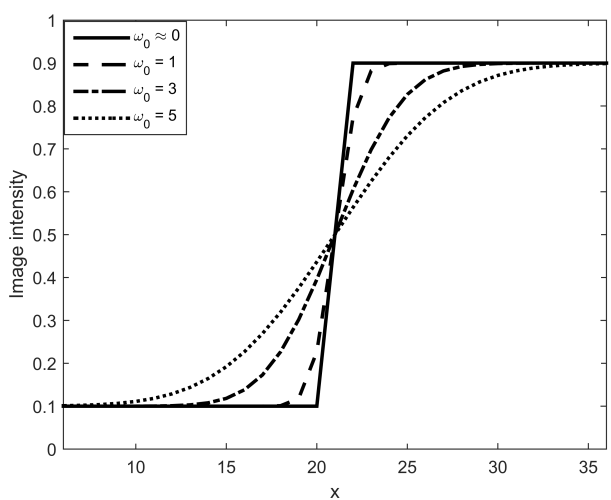

Fig. 2: The horizontal intensity profile through the center of the image shown in Fig. 1(a), 1(b), 1(c) and 1(d), respectively.

\subsection{Normalization for FDAG kernels}

In order to see whether or not the directional Canny kernel can be used in scale-space directly, we compute its theoretical convolutional response to a scaled edge. Considering the ideal case that the Canny kernel is oriented normal to the direction of the observed edge, i.e., $\theta=\theta_{0}$, from Eqs. (9) and (13), we obtain the ideal Canny edge strength at the center of the edge:

$$
\begin{aligned}
E_{\mathrm{C}} & =\max _{\theta}\left|f\left(\mathbf{x} ; \omega_{0}, \theta_{0}\right) * g_{\mathrm{C}}^{\prime}(\mathbf{x} ; \sigma, \theta)\right| \\
& =\sqrt{2 \pi} c_{0} \frac{\sigma^{2}}{\sqrt{\omega_{0}^{2}+\sigma^{2}}} .
\end{aligned}
$$

Obviously, $E_{\mathrm{C}}$ is a monotonically increasing function of $\sigma$. That is, the obtained edge strength increases as the kernel scale increases. This makes it impossible to identify the scale of the edge using the Canny kernel. Moreover, the choice of the scale for the Canny kernel has a great impact on the obtained edge strength. As a conclusion, the Canny kernel cannot be applied in scale-space directly.

To study the performance of the traditional non-normalized FDAG kernels $[38,50]$ in scale-space, from Eqs. (12) and (13), we compute the edge strength in scale-space as fol- 
lows:

$$
\begin{aligned}
E_{\text {non }} & =\max _{\theta}\left|f\left(\mathbf{x} ; \omega_{0}, \theta_{0}\right) * g^{\prime}(\mathbf{x} ; \sigma, \varphi, \theta)\right| \\
& =\frac{c_{0}}{\sqrt{2 \pi\left(\omega_{0}^{2}+\sigma^{2}\right)}} .
\end{aligned}
$$

Note that the edge strength in Eq. (17) is independent of the anisotropy factor $\varphi$. Unfortunately, $E_{\text {non }}$ is a monotonically decreasing function of $\sigma$. That is, the obtained edge strength decreases as the kernel scale increases. Therefore, the non-normalized FDAG kernels cannot be applied in scale-space directly either.

According to the analysis above, we learn that neither the Canny kernels nor the nonnormalized FDAG kernels can identify the scale of the observed edge. To solve the scaling problem for FDAG kernels, we need a novel normalization method. Inspired by Lindeberg's $\gamma$-parameterized normalization in Eq. (3), we normalize the FDAG kernel as follows:

$$
\tilde{g}^{\prime}(\mathbf{x} ; \sigma, \varphi, \theta)=\beta \sigma^{2 \gamma} \cdot g^{\prime}(\mathbf{x} ; \sigma, \varphi, \theta),
$$

where $\beta$ is a factor that controls the extent of the filtering response. Hence, in the ideal case, i.e., $\theta=\theta_{0}$, the edge strength obtained by the normalized FDAG kernel in scale-space is

$$
\begin{aligned}
E_{\text {norm }} & =\max _{\theta}\left|f\left(\mathbf{x} ; \omega_{0}, \theta_{0}\right) * \tilde{g}^{\prime}(\mathbf{x} ; \sigma, \varphi, \theta)\right| \\
& =\beta c_{0} \frac{\sigma^{2 \gamma}}{\sqrt{2 \pi\left(\omega_{0}^{2}+\sigma^{2}\right)}} .
\end{aligned}
$$

To study the monotonicity of $E_{\text {norm }}$ in scale-space, we compute the derivative of $E_{\text {norm }}$ w.r.t. $\sigma$ :

$$
\frac{\partial E_{\mathrm{norm}}}{\partial \sigma}=\frac{\sqrt{2 \pi}}{\pi} \beta c_{0} \gamma \sigma^{2 \gamma-1}\left(\omega_{0}^{2}+\sigma^{2}\right)^{-\frac{1}{2}}-\frac{\sqrt{2 \pi}}{2 \pi} \beta c_{0} \sigma^{2 \gamma+1}\left(\omega_{0}^{2}+\sigma^{2}\right)^{-\frac{3}{2}} .
$$

For $\sigma \in \mathbb{R}_{+}$, it is easy to verify that the second-order derivative of $E_{\text {norm }}$ w.r.t. $\sigma$ is negative. Therefore, by setting the partial derivative in Eq. (20) to zero, we find that $E_{\text {norm }}$ reaches its maximum value at the scale

$$
\sigma^{*}=\sqrt{\frac{2 \gamma}{1-2 \gamma}} \omega_{0}
$$

Since $\sigma \in \mathbb{R}_{+}$, we have $\left.\gamma \in\right] 0,0.5\left[\right.$. To make $E_{\text {norm }}$ reach its maximum value at the original edge scale, i.e., $\sigma^{*}=\omega_{0}$, we set $\gamma=0.25$. Accordingly, the edge strength in Eq. (19) becomes

$$
E_{\text {norm }}^{*}=\frac{\beta c_{0}}{2 \sqrt{\pi \omega_{0}}} .
$$

Based on Eq. (22), $\beta$ should be set as $\beta=2 \sqrt{\pi \omega_{0}}$ to make $E_{\text {norm }}^{*}$ equal $c_{0}$, because in this case, the obtained edge strength would precisely reflect the true edge strength. However, the parameter $\omega_{0}$, i.e., the scale of the observed edge, is unknown beforehand. Fortunately, from Eqs. (20) and (21), the maximum edge strength value in scale-space and the scale 
identification procedure are both independent of the argument $\beta$. Here, we set $\beta=2 \sqrt{\pi}$ at first, and accordingly the edge strength in Eq. (22) becomes

$$
E_{\text {norm }}^{*}=\frac{c_{0}}{\sqrt{\omega_{0}}}
$$

Once the edge scale $\omega_{0}$ is identified, the edge strength will be compensated by a multiplication factor $\sqrt{\omega_{0}}$ to make the obtained edge strength equal the true edge strength $c_{0}$. The compensation procedure will be elaborated later in Section 5. Note that in Eq. (23), $E_{\text {norm }}^{*}$ does not depend on the anisotropy factor $\varphi$, which means that the choice of $\varphi$ will not affect the measure of edge strength in a noise-free case.

Hence, having $\beta=2 \sqrt{\pi}$ and $\gamma=0.25$, from Eq. (18), the normalized FDAG kernel is given by:

$$
\tilde{g}^{\prime}(\mathbf{x} ; \sigma, \varphi, \theta)=2 \sqrt{\pi} \sigma^{\frac{1}{2}} \cdot g^{\prime}(\mathbf{x} ; \sigma, \varphi, \theta)
$$

Consequently, using a family of normalized FDAG kernels covering all possible scales and orientations, we are able to identify the scales of the observed edges while obtaining the edge strength maps in scale-space.

\section{Alleviation of the anisotropy stretch effect}

The problem of the anisotropy stretch effect, as mentioned in the previous section, still remains unsolved. In order to obtain a good noise-robustness and to alleviate the anisotropy stretch effect, based on an SNR analysis, we propose an adaptive anisotropy factor for the MFDAG kernels in this section.

\subsection{SNR analysis}

In the signal processing field, the SNR has been widely used to analyze the capability of a filter to suppress noise [17]. It is defined as the quotient of the maximum signal response and the standard deviation of the filtered noise [29]. Here, we compare the monoscale and isotropic Canny kernel with the MFDAG kernels in terms of the SNR.

As mentioned earlier, due to the trade-off between a good localization and a good noiserobustness, the scale selection in the Canny method is usually a dilemma [38]. On the one hand, to guarantee a good localization, we prefer to choose a scale as small as possible for the Canny kernel. On the other hand, to obtain a good robustness to noise, we have to choose a scale that is large enough. Therefore, the scale of the Canny kernel has to be selected carefully. In contrast to the monoscale framework, a multiscale framework can ease the scale selection. Without loss of generality, we set the scale of the Canny kernel as $\sigma_{1}$ while configuring the scale set of the MFDAG kernels as $\mathbb{S}=\left\{\sigma \mid \sigma_{1} \leq \sigma \leq \sigma_{2}\right\}$.

Suppose the image to be processed is corrupted by zero-mean white Gaussian noise $\xi(\mathbf{x})$ with variance $\varepsilon_{0}^{2}$. The SNR analysis of the directional Canny kernel and the MFDAG kernels are presented as follows.

For the directional Canny kernel, the edge strength is obtained by filtering the image using Eq. (9). As a result, the intensity of noise filtered by the directional Canny kernel is 
given by [5]:

$$
\begin{aligned}
\varepsilon_{\mathrm{C}} & =\left.\varepsilon_{0} \sqrt{\iint_{\mathbb{R}^{2}}\left(g_{\mathrm{C}}^{\prime}(\mathbf{x} ; \sigma, \theta)\right)^{2} \mathrm{~d} \mathbf{x}}\right|_{\sigma=\sigma_{1}, \theta=\theta_{0}} \\
& =\frac{\sqrt{2 \pi} \varepsilon_{0}}{2} .
\end{aligned}
$$

From Eqs. (16) and (25), we compute the SNR of the directional Canny kernel as:

$$
\begin{aligned}
\operatorname{SNR}_{\mathrm{C}} & =\left.\frac{E_{\mathrm{C}}}{\varepsilon_{\mathrm{C}}}\right|_{\sigma=\sigma_{1}} \\
& =\frac{2 c_{0} \sigma_{1}^{2}}{\varepsilon_{0} \sqrt{\sigma_{1}^{2}+\omega_{0}^{2}}} .
\end{aligned}
$$

With respect to the MFDAG kernels, we compute the intensity of noise filtered by a normalized FDAG kernel at the scale $\sigma$ as follows:

$$
\begin{aligned}
\varepsilon_{\text {norm }} & =\left.\varepsilon_{0} \sqrt{\iint_{\mathbb{R}^{2}}\left(\tilde{g}^{\prime}(\mathbf{x} ; \sigma, \varphi, \theta)\right)^{2} \mathrm{~d} \mathbf{x}}\right|_{\theta=\theta_{0}} \\
& =\frac{\varepsilon_{0}}{\sigma \sqrt{2 \varphi \sigma}} .
\end{aligned}
$$

From Eq. (27), one can see that $\varepsilon_{\text {norm }}$ has an inverse relationship with the kernel scale $\sigma$ and the anisotropy factor $\varphi$. Thus, for a given scale set $\mathbb{S}=\left\{\sigma \mid \sigma_{1} \leq \sigma \leq \sigma_{2}\right\}$, if the maximum aggregation scheme is employed, kernels with smaller scales tend to produce larger noise responses, which thereby are more likely to be selected as the final edge strength. In other words, the intensity of noise in the edge strength is mainly determined by the small-scale kernels.

Next, the SNR obtained by the MFDAG kernels is analyzed in two situations. Firstly, in the case $\omega_{0} \leq \sigma_{1}$, according to the monotonicity of $E_{\text {norm }}$, the MFDAG kernels yield the maximum edge strength at the scale $\sigma_{1}$. Thus, in terms of Eqs. (19) and (27), the SNR of the MFDAG kernels is given by:

$$
\begin{aligned}
\mathrm{SNR}_{\text {norm }} & =\left.\frac{E_{\text {norm }}}{\varepsilon_{\text {norm }}}\right|_{\sigma=\sigma_{1}} \\
& =\frac{2 c_{0} \sigma_{1}^{2} \sqrt{\varphi}}{\varepsilon_{0} \sqrt{\sigma_{1}^{2}+\omega_{0}^{2}}} .
\end{aligned}
$$

Secondly, if the edge scale $\omega_{0}$ is within the interval $\left[\sigma_{1}, \sigma_{2}\right]$, we get the maximum edge strength at the scale $\sigma^{*}=\omega_{0}$. In terms of Eqs. (23) and (27), the obtained SNR is computed as:

$$
\begin{aligned}
\mathrm{SNR}_{\text {norm }}^{*} & =\left.\frac{E_{\text {norm }}^{*}}{\varepsilon_{\text {norm }}}\right|_{\sigma=\omega_{0}} \\
& =\frac{2 c_{0} \sigma_{1}^{2} \sqrt{\varphi}}{\varepsilon_{0} \sqrt{2 \omega_{0} \sigma_{1}}} .
\end{aligned}
$$


From Eqs. (26), (28) and (29), it is easy to verify that

$$
\mathrm{SNR}_{\text {norm }}^{*} \geq \mathrm{SNR}_{\text {norm }} \geq \mathrm{SNR}_{\mathrm{C}} .
$$

Therefore, we arrive at the conclusion that the proposed MFDAG kernels are more robust to noise than the isotropic Canny kernel.

\subsection{Adaptive anisotropy factor}

It has been reported that the FDAG kernel incurs an anisotropy stretch effect, which subsequently would lead to spurious detection results [38]. As mentioned earlier, this is due to the fact that, for an FDAG kernel, the blurring extent w.r.t. $x$ is determined by $\sigma$, while the blurring extent w.r.t. $y$ is determined by $\varphi \sigma$. In this paper, we present a method to alleviate the anisotropy stretch effect by using an adaptive anisotropy factor.

From Eq. (19), we learn that $E_{\text {norm }}$ is independent of the anisotropy factor $\varphi$. Also, from Eqs. (27), (28) and (29), we find that the intensity of noise in the obtained edge strength is mainly determined by the small-scale kernels. In other words, it is not necessary to apply anisotropy factors to all the scales, especially to large scales, since the latter can ensure a good noise-robustness even if the anisotropy of the kernel is absent. Hence, in order to alleviate the anisotropy stretch effect while retaining a good noise-robustness, specifying the kernel scale set as $\mathbb{S}=\left\{\sigma \mid \sigma_{1} \leq \sigma \leq \sigma_{2}\right\}$, we propose the adaptive anisotropy factor as follows:

$$
\varphi(\sigma)= \begin{cases}\frac{\sigma_{\mathrm{con}}^{2}}{\sigma^{2}} & , \text { if } \sigma_{1} \leq \sigma \leq \sigma_{\mathrm{con}} \\ 1 & , \text { otherwise }\end{cases}
$$

where $\sigma_{\text {con }} \in\left[\sigma_{1}, \sigma_{2}\right]$ stands for the robustness control scale. More specifically, when we use kernels with $\sigma \leq \sigma_{\text {con }}$, we introduce a large anisotropy factor to improve the noise robustness. When we use a kernel with a large scale, i.e., $\sigma>\sigma_{\text {con }}$, the anisotropy factor is set as $\varphi=1$, because a large $\sigma$ is already able to guarantee a good noise-robustness.

By substituting Eq. (31) into Eqs. (28) and (29), we have

$$
\begin{aligned}
\mathrm{SNR}_{\mathrm{norm}} & =\frac{2 c_{0} \sigma_{1} \sigma_{\mathrm{con}}}{\varepsilon_{0} \sqrt{\sigma_{1}^{2}+\omega_{0}^{2}}} \\
\mathrm{SNR}_{\text {norm }}^{*} & =\frac{2 c_{0} \sigma_{1} \sigma_{\mathrm{con}}}{\varepsilon_{0} \sqrt{2 \omega_{0} \sigma_{1}}} .
\end{aligned}
$$

One can see that once the scale set $\mathbb{S}$ is given, the SNR of the edge strength can be controlled by $\sigma_{\text {con }}$. Furthermore, comparing Eq. (32) to Eq. (26), given $\sigma_{\text {con }} \geq \sigma_{1}$, we still have $\mathrm{SNR}_{\text {norm }}^{*} \geq \mathrm{SNR}_{\text {norm }} \geq \mathrm{SNR}_{\mathrm{C}}$.

As for the setting of the control scale, we generally recommend to set $\sigma_{\mathrm{con}}$ as the geometric mean of $\sigma_{1}$ and $\sigma_{2}$, because in this case the blurring extent at each scale is determined by $\sigma \varphi$, which is given by

$$
\sigma \varphi=\sigma \cdot \frac{\sigma_{\mathrm{con}}^{2}}{\sigma^{2}}=\frac{\sigma_{1} \sigma_{2}}{\sigma}
$$

Since $\sigma_{1} \leq \sigma \leq \sigma_{2}$, it holds that $\sigma \varphi \leq \sigma_{2}$, which means that in the resulting multiscale edge strength, the blurring extent over all the directions would be mainly determined by $\sigma_{2}$. 
Consequently, the anisotropy stretch effect can be alleviated by using an adaptive anisotropy factor.

Eventually, by substituting Eq. (31) into Eqs. (12) and (24), we get MFDAG kernels with an adaptive anisotropy factor.

\section{Edge detection using MFDAG kernels}

In this section, we firstly present the discrete MFDAG kernels. Secondly, we study a compensation procedure for the obtained edge strength map, which is mentioned in Section 3. Eventually, by incorporating a binarization procedure, we develop a full-fledged method for multiscale edge detection.

\subsection{Discrete filter bank}

In order to accommodate the proposed normalized FDAG kernels to digital image processing, discrete versions of these kernels are needed. We obtain both the discrete Gaussian kernel and the discrete normalized FDAG kernel by sampling the formulae in Eqs. (11), (12) and (24) in the $2 \mathrm{D}$ integer coordinate $\mathbb{Z}^{2}$ :

$$
\begin{aligned}
g\left(\mathbf{m} ; \sigma_{i}, \varphi_{i}, \theta_{j}\right) & =\frac{1}{2 \pi \varphi_{i} \sigma_{i}^{2}} \exp \left(-\frac{1}{2 \sigma_{i}^{2}} \mathbf{m}^{T} \mathbf{R}_{j}^{T}\left[\begin{array}{cc}
1 & 0 \\
0 & \varphi_{i}^{-2}
\end{array}\right] \mathbf{R}_{j} \mathbf{m}\right) \\
\tilde{g}^{\prime}\left(\mathbf{m} ; \sigma_{i}, \varphi_{i}, \theta_{j}\right) & =-2 \sqrt{\pi} \sigma_{i}^{\frac{1}{2}} \cdot \frac{\left[\cos \theta_{j}, \sin \theta_{j}\right] \mathbf{m}}{\sigma_{i}^{2}} g\left(\mathbf{m} ; \sigma_{i}, \varphi_{i}, \theta_{j}\right),
\end{aligned}
$$

where

$$
\begin{aligned}
\varphi_{i} & =\max \left(\frac{\sigma_{\mathrm{con}}^{2}}{\sigma_{i}^{2}}, 1\right), \\
\mathbf{R}_{j} & =\left[\begin{array}{rr}
\cos \theta_{j} & \sin \theta_{j} \\
-\sin \theta_{j} & \cos \theta_{j}
\end{array}\right],
\end{aligned}
$$

$\mathbf{m}=\left[m_{x}, m_{y}\right]^{T} \in \mathbb{Z}^{2}$ represents the image coordinates, $\sigma_{i} \in \mathbb{S}, \theta_{j} \in \mathbb{D}$ and $\varphi_{i}$ denote the scale, orientation and adaptive anisotropy factor, respectively, while $\mathbb{S}$ and $\mathbb{D}$ denote the scale set and orientation set, respectively. These discrete normalized FDAG kernels form the discrete MFDAG kernels in scale-space. Examples of discrete normalized FDAG kernels are illustrated in Fig. 3.

Natural images have either one channel (grayscale images) or three channels (color images). Let a 2D image signal $I^{k}(\mathbf{m})$ denote the $k$-th channel of a given image $I(\mathbf{m})$. A filter bank of discrete normalized FDAG kernels covering all the possible scales and orientations is employed to convolve each channel of the image:

$$
\mathscr{E}^{k}\left(\mathbf{m} ; \sigma_{i}, \varphi_{i}, \theta_{j}\right)=\left|I^{k}(\mathbf{m}) * \tilde{g}^{\prime}\left(\mathbf{m} ; \sigma_{i}, \varphi_{i}, \theta_{j}\right)\right| .
$$

Note that $k=1$ for grayscale images while $k \in\{1,2,3\}$ for color images.

At each location, by employing the maximum aggregation scheme in scale-space for each channel and, subsequently, by selecting the maximum value of the edge strength over all the channels $[14,42]$, we obtain the maximum response map by

$$
\mathscr{E}(\mathbf{m})=\max _{k} \max _{\sigma_{i} \in \mathbb{S}} \max _{\theta_{j} \in \mathbb{D}} \mathscr{E}^{k}\left(\mathbf{m} ; \sigma_{i}, \varphi_{i}, \theta_{j}\right) .
$$




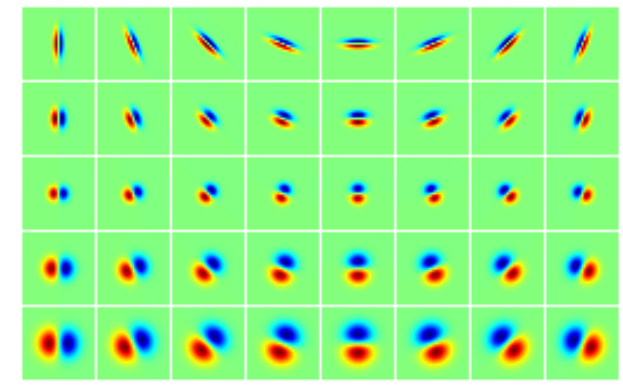

Fig. 3: Examples of discrete normalized FDAG kernels. The control scale is set as $\sigma_{\text {con }}=2$. Top row: Kernels with $\sigma=1$ and $\varphi=4$. Second row: Kernels with $\sigma=1.5$ and $\varphi=1.78$. Third row: Kernels with $\sigma=2$ and $\varphi=1$. Fourth row: Kernels with $\sigma=3$ and $\varphi=1$. Bottom row: Kernels with $\sigma=4$ and $\varphi=1$. The intensity range of each patch has been adjusted for better display.

As an illustration, Fig. 4(c) shows the resulting maximum response map obtained on Fig. 4(a). In contrast, Fig. 4(b) shows an edge strength map obtained at a single scale. Obviously, Fig. 4(c) has a better representation for the spatially scaled edges than Fig. 4(b).

From Eqs. (20), (21) and (22), we learn that the response reaches its maximum value at the scale of the original edge scale, and as such, at each pixel position, the scale map is obtained by maximizing $\mathscr{E}^{k}\left(\mathbf{m} ; \sigma_{i}, \varphi_{i}, \theta_{j}\right)$ in scale-space. More specifically, we obtain the scale map $S(\mathbf{m})$ by

$$
S(\mathbf{m})=\underset{\sigma_{i} \in \mathbb{S}}{\arg \max } \max _{k} \max _{\theta_{j} \in \mathbb{D}} \mathscr{E}^{k}\left(\mathbf{m} ; \sigma_{i}, \varphi_{i}, \theta_{j}\right) .
$$

In addition, the edge direction map $D(\mathbf{m})$ is obtained by maximizing the edge strength as follows:

$$
D(\mathbf{m})=\underset{\theta_{j} \in \mathbb{D}}{\arg \max } \max _{k} \max _{\sigma_{i} \in \mathbb{S}} \mathscr{E}^{k}\left(\mathbf{m} ; \sigma_{i}, \varphi_{i}, \theta_{j}\right)
$$

\subsection{Compensated edge strength}

As mentioned in Section 3, according to Eq. (23), there is a bias in the edge strength obtained by Eq. (37). Fortunately, this bias can be compensated once the edge scale is identified. In this paper, we recommend a scale-based compensation procedure for the edge strength map, especially in the scenario that many spatially scaled edges occur in the image.

In practice, an edge detection result is usually represented by an edge map consisting of curved centerlines (one pixel wide) of edges. The candidate pixels of edge centerlines can be identified by the technique of nonmaxima suppression (NMS) [36]. Thus, for the sake of computational efficiency, there is no need to compensate the edge strength at all the positions. In other words, it suffices to only compensate the edge strength at positions of candidates of edge centerlines. According to Eq. (23), the compensation factor is determined by the square root of the identified scale. Denoting the result of the NMS procedure as $\mathscr{E}_{\text {nms }}(\mathbf{m})$, we obtain the compensated edge strength map as

$$
\mathscr{E}_{\mathrm{ce}}(\mathbf{m})=\mathscr{E}_{\mathrm{nms}}(\mathbf{m}) \cdot \sqrt{S(\mathbf{m})}
$$




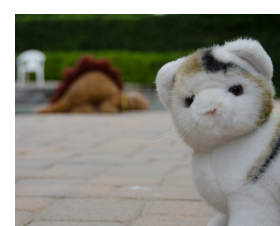

(a)

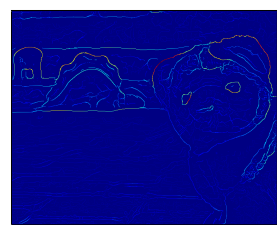

(e)

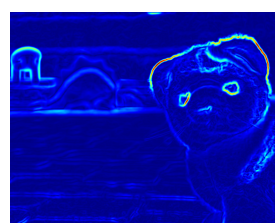

(b)

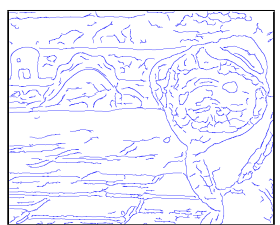

(f)

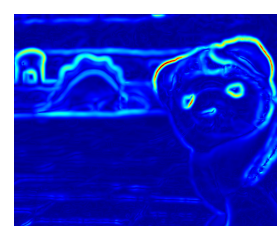

(c)

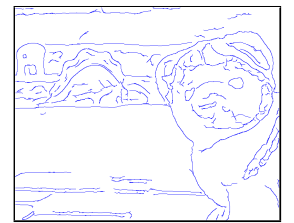

(g)

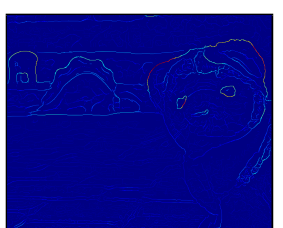

(d)

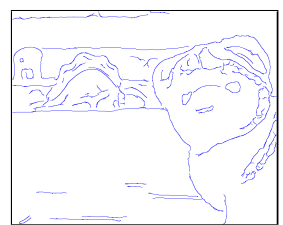

(h)

Fig. 4: Illustration of the edge detection process. (a) The original image (courtesy of Dave Johnson); (b) Edge strength map obtained at a single scale; (c) The maximum response map; (d) The NMS result; (e) The compensated edge strength map; (f)-(h) The binarization results using an upper threshold of $0.05,0.10$ and 0.15 , respectively.

As an illustration, the NMS result of Fig. 4(c) is shown in Fig. 4(d), while the compensated edge strength map is shown in Fig. 4(e). Comparing Fig. 4(e) to Fig. 4(d), one can easily see that the edge strength of some spatially scaled edges has been enhanced by the compensation procedure.

\subsection{Binarization}

Having obtained the edge strength map, we next require a binarization procedure to segment and thin the edge strength map into a binary edge map. As briefly mentioned in Section 1, quite a few techniques have been proposed [33] to implement the binarization procedure. Since this paper mainly addresses the measurement of the edge strength, we straightforwardly adopt the widely used hysteresis segmentation $[5,39,42,50]$ to produce the binary edge map.

Hysteresis segmentation is a double-thresholding process [5]. It employs an upper threshold $\tau_{1}$ as well as a lower threshold $\tau_{2}=\tau_{1} / \kappa$, where the ratio $\kappa \in[2,3]$. Here, we empirically set this ratio as $\kappa=2$. Mostly, hysteresis segmentation is realized in two steps. Given an edge strength map processed by the NMS procedure, all the locations at which the edge strength exceeds the upper threshold are first labelled as edge pixels. The locations at which the edge strength is between the upper threshold and the lower threshold are labelled as potential edge pixels. For each of these potential edge pixels, if there is a path to connect it with an edge pixel, it is also marked as an edge pixel. Subsequently, all these edge pixels form the binary detection result $\mathscr{E}_{\mathrm{d}}(\mathbf{m})$. In Figs. 4(f), 4(g) and 4(h), we illustrate three edge maps obtained by three different upper thresholds.

Eventually, as illustrated in Fig. 5, a full-fledged framework for edge detection is developed. 


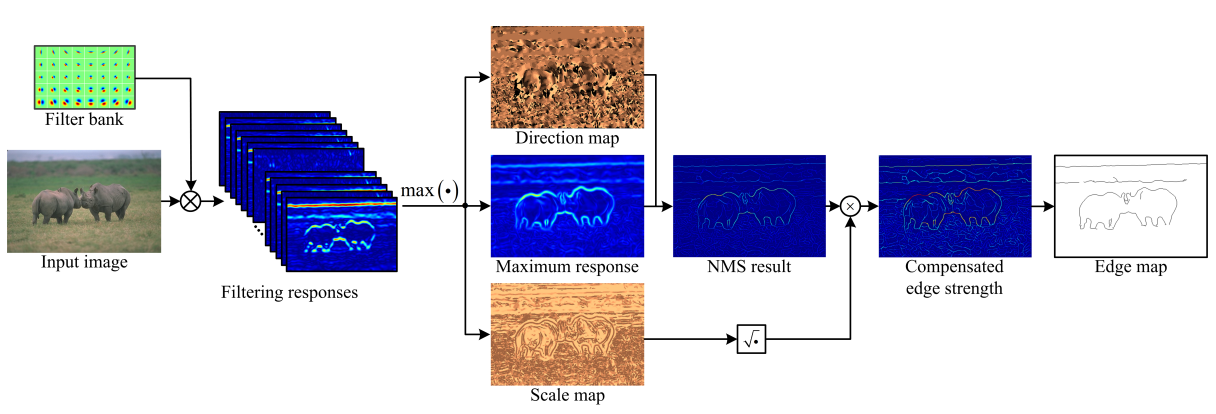

Fig. 5: The flowchart of our framework for multiscale edge detection.

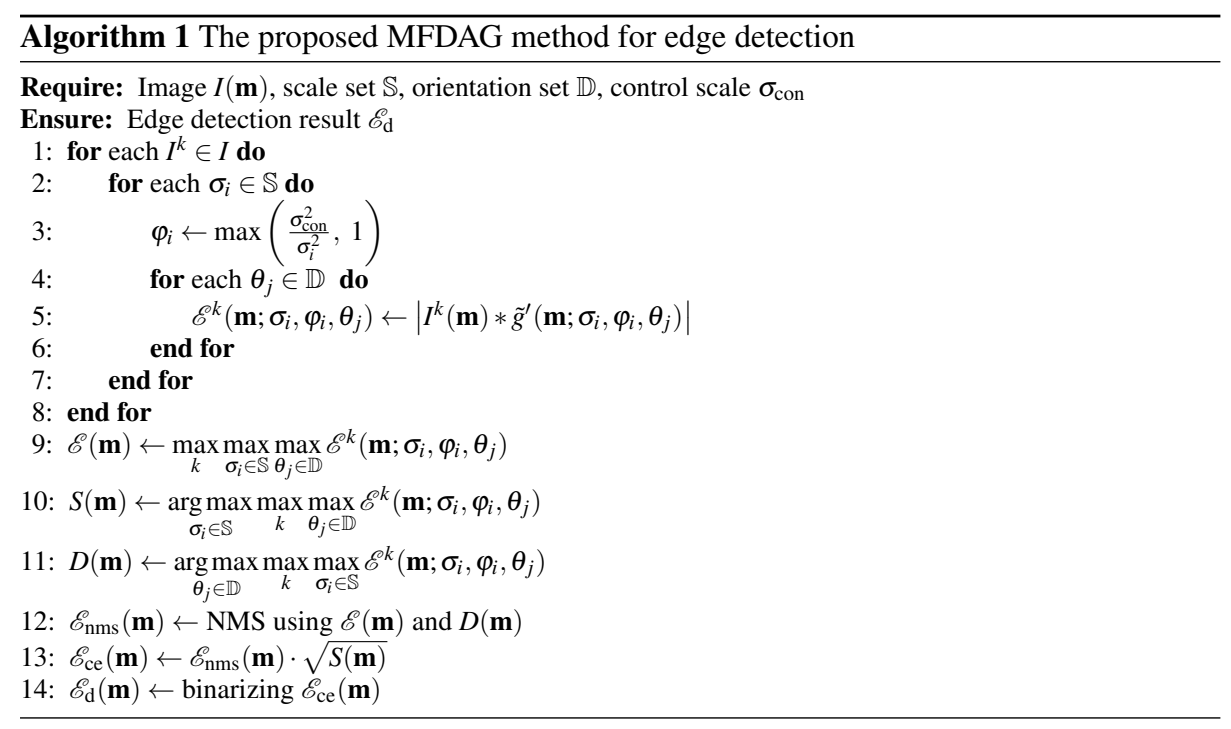

\section{Experimental validation}

In this paper, we have proposed an edge detection method (MFDAG method) using the MFDAG kernels. Now we are in a position to validate whether the proposed method can yield better edge detection results, and accordingly facilitate practical tasks. In practice, a quantitative performance evaluation of an edge detector should hinge on a specific application [23]. Like many edge detection studies [12,30,49,24], we evaluate the proposed method, which is summarized in Algorithm 1, on the Rijksuniversiteit Groningen (RuG40) image dataset [11] and the Berkeley Segmentation Data Set and benchmarks 500 (BSDS500) [28]. To make the evaluation more convincing, our method will also be compared with the competing methods.

\subsection{Evaluation metrics}

Given an edge detection result and its corresponding ground truth edge map(s), an evaluation procedure is needed to determine how well the detection result approximates the ground 
truth. Since edge detection can be considered as a binary classification procedure discriminating edge pixels form non-edge pixels, the comparison between the detection result and the ground truth can be formulated in terms of success and failure. In this respect, the performance can be evaluated by the precision-recall framework. Precision is the probability that a detected edge pixel is a true edge pixel, while recall is the probability that a true edge pixel is successfully detected. In practice, we carry out the performance evaluation using the paradigm proposed by Martin [27], which is also widely adopted in literature [1,8].

Let $\mathscr{E}_{\mathrm{d}}$ be an edge detection result and let $\mathscr{G}=\left\{\mathscr{G}^{1}, \mathscr{G}^{2}, \ldots, \mathscr{G}^{N_{\mathrm{G}}}\right\}$ be its corresponding ground truth edge map(s), where $N_{\mathrm{G}} \in \mathbb{N}_{+}$denotes the total number of ground truth edge map(s). The Cost Scaling Assignment (CSA) algorithm [10] is employed to perform a pixelto-pixel matching between $\mathscr{E}_{\mathrm{d}}$ and each of the ground truth edge maps in $\mathscr{G}$. On the one hand, for each detected edge pixel in $\mathscr{E}_{\mathrm{d}}$, if it matches any of the ground truth edge pixels in $\mathscr{G}$ within a spatial tolerance distance, this detected edge pixel is considered as a true positive detection pixel; otherwise, it is considered as a false positive detection pixel. On the other hand, for each true edge pixel in each ground truth edge map, if it is matched by a detected edge pixel in $\mathscr{E}_{\mathrm{d}}$ within a spatial tolerance distance, this ground truth edge pixel is counted as a matched ground truth pixel; otherwise, it is counted as an unmatched ground truth pixel. These matched ground truth pixels and unmatched ground truth pixels in all the ground truth map(s) compose the aggregate matched ground truth pixels and aggregate unmatched ground truth pixels, respectively. In this way, the precision and recall are computed by:

$$
\mathrm{PREC}=\frac{\mathrm{TP}}{\mathrm{TP}+\mathrm{FP}} \quad \text { and } \quad \mathrm{REC}=\frac{\mathrm{MT}}{\mathrm{MT}+\mathrm{UM}}
$$

where TP, FP, MT and UM are the numbers of true positive detection pixels, false positive detection pixels, aggregate matched ground truth pixels and aggregate unmatched ground truth pixels, respectively. Different thresholds lead to different detection results, thus resulting different PREC-REC pairs, which can subsequently be interpolated to form a PrecisionRecall (PR) curve. For two PR curves, the curve that is farther from the origin is preferred. That is, a curve that has larger area under the PR curve indicates a better detection performance [1]. The area under the PR curve can also be measured by the Average Precision (AP) [8]. A deeper insight in evaluation paradigms can be found in [23,22].

To determine the optimal threshold for an edge detector, the $F$-measure, which computes the harmonic mean of precision and recall as expressed in Eq. (42), is used as an evaluation measure:

$$
F=\frac{2 \cdot \mathrm{PREC} \cdot \mathrm{REC}}{\mathrm{PREC}+\mathrm{REC}}
$$

There are two ways to determine the optimal threshold in terms of the $F$-measure. The first way is to apply an identical threshold to all the edge strength maps, and accordingly the threshold yielding the largest $F$-measure $\left(F_{\mathrm{ODS}}\right)$ is named as the optimal dataset scale (ODS) threshold. The second way is to apply a separate threshold to the edge strength maps one by one, and for each edge strength map, the threshold yielding the largest $F$-measure is selected to form the optimal image scale (OIS) thresholds. Accordingly, we can obtain the OIS $F$-measure ( $F_{\mathrm{OIS}}$ ) using the OIS thresholds.

We also adopt the evaluation measure Recall at 50\% Precision (R50), which reflects the detection accuracy when the recall is high [8]. 


\subsection{Experimental setup}

To make the evaluation more convincing, we compare our method with several competing methods, including the Canny method [5], the color variant of the Canny method (CVC method) [14], the method based on isotropic and anisotropic Gaussian kernels (IAGK method) [38], the method based on a gradient matrix with anisotropic Gaussian direction derivatives (GMAGDD method) [42], the method based on automatic anisotropic Gaussian kernels (AAGK method) [50] and the method using self-adapting weighted operators (SAWO method) [24]. Note that the SAWO method is only applicable for grayscale images, while the CVC and GM-AGDD methods are only applicable for color images. All the competing methods are differentiation-based and Gaussian-based methods, representing different strategies of measuring the edge strength. To highlight the impact of different strategies on edge strength measurement, the binarization procedure is kept identical for all the methods.

In this experimental validation section, each method has been configured as follows:

- Canny: The scale is set to be $\sqrt{2}$, which is widely adopted in literature $[38,50,37]$.

- CVC: The method is implemented in the RGB color space [14] and the scale is set to be $\sqrt{2}$.

- IAGK: According to the original setting [38], the scale is specified as 4; the anisotropy factor is set to be $2 \sqrt{2}$ and the number of kernel orientations is set to be 16 .

- GM-AGDD: The scale is set to be 6; the anisotropy factor is specified as 6 and the number of kernel orientations is set to be 16 . These parameter settings are originally recommended [42].

- AAGK: The number of kernel orientations is originally set to be 8 [50], and accordingly, the scale and the anisotropy factor are computed as $\sqrt{10}$ and $\sqrt{5}$, respectively.

- SAWO: According to [24], the scale set is configured as $\mathbb{S}=\{1.0+0.5 \cdot(i-1) \mid i \in$ $1,2, \ldots, 6\}$. The homogeneously-weighted operator $\left(\mathbf{h}=\left(0,0, \frac{1}{3}, \frac{1}{3}, \frac{1}{3}, 0\right)\right)$ is used to fuse the multiple gradients.

- MFDAG: The scale set is configured with $\mathbb{S}=\{2+0.2 \cdot(i-1) \mid i \in 1,2, \ldots, 11\}$, while the control scale and the number of kernel orientations are set to be $2 \sqrt{2}$ and 8 , respectively. For color images, we use the CIE Lab color space.

Furthermore, in the binarization procedure, the upper threshold is specified with values from 0.01 to 0.99 with a step of 0.01 . The matching tolerance distance in the evaluation procedure is set to be $0.75 \%$ of the image diagonal $[1,21,47]$.

\subsection{Results on the RuG40 dataset}

We first evaluate the proposed MFDAG method on the RuG40 dataset ${ }^{1}$, which contains 40 grayscale images accompanied by the ground truth edge maps. These ground truth edge maps are labelled by a single annotator [11]. Sample images as well as the ground truth edge maps are illustrated in Fig. 6. We also compare our method with four competing methods, i.e., the Canny [5], IAGK [38], AAGK [50] and SAWO [24] methods, reporting both quantitative and qualitative evaluation results below.

The resulting PR curves are shown in Fig. 7, while the results of evaluation measures are reported in Tab. 1. One can see that the PR curve of the MFDAG method is farther from the origin than for the competing methods. This is consistent with the evaluation result in

\footnotetext{
1 http://www.cs.rug.nl/ imaging/databases/contour_database/contour_database
} 


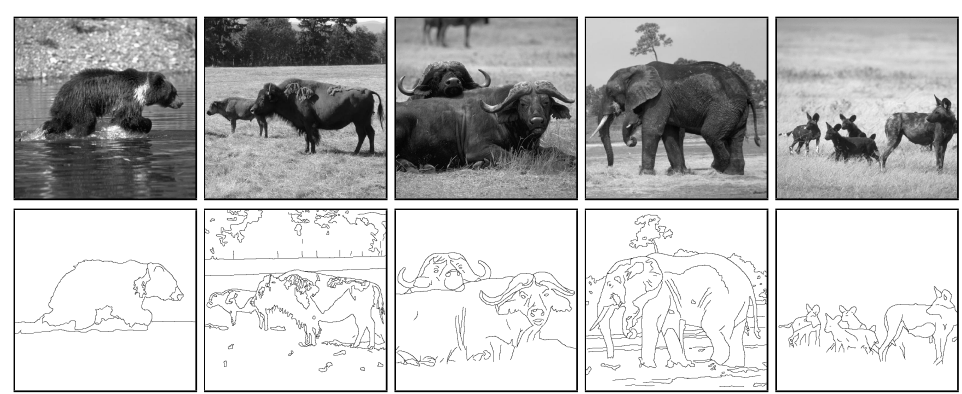

Fig. 6: Sample images and the ground truth edge maps from the RuG40 dataset. First row: Original images. Second row: The ground truth edge maps.

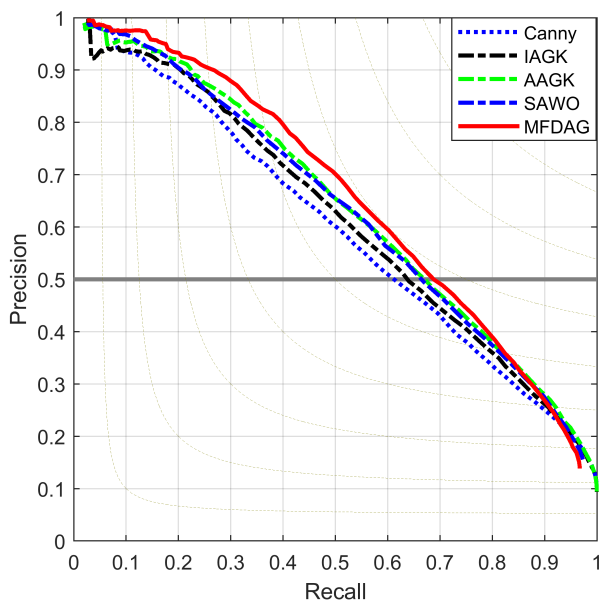

Fig. 7: PR curves of different methods obtained on the RuG40 dataset.

terms of AP. In comparison to other methods, our method also yields larger $F_{\mathrm{ODS}}$ and $F_{\mathrm{OIS}}$ values. This manifests that the MFDAG method makes a better trade-off between precision and recall over the competing methods. Besides, our method obtains the largest R50 value, which demonstrates that our method is more effective in the high recall regime than the competing methods.

Samples of detection results shown in Fig. 8 also confirm the superiority of our method. Compared with the other methods, our method yields more authentic edges, while showing less spurious edges. In particular, the MFDAG method improves the detection of spatially scaled edges. For example, in the image shown in the third row of Fig. 8, there are spatially scaled edges in the background. While the Canny, IAGK, AAGK and SAWO methods fail in detecting some blurred edges, the MFDAG method depicts most of the spatially scaled edges successfully.

The above experimental results demonstrate that the MFDAG method outperforms all the competing methods on the RuG40 dataset. 
Table 1: Results of evaluation measures obtained by different methods on the RuG40 dataset.

\begin{tabular}{lcccc}
\hline Methods & $F_{\text {ODS }}$ & $F_{\text {OIS }}$ & AP & R50 \\
\hline Canny & 0.55 & 0.65 & 0.59 & 0.61 \\
IAGK & 0.57 & 0.68 & 0.61 & 0.64 \\
AAGK & 0.58 & 0.69 & 0.64 & 0.67 \\
SAWO & 0.58 & 0.67 & 0.64 & 0.66 \\
MFDAG & $\mathbf{0 . 6 0}$ & $\mathbf{0 . 7 0}$ & $\mathbf{0 . 6 7}$ & $\mathbf{0 . 6 9}$ \\
\hline
\end{tabular}

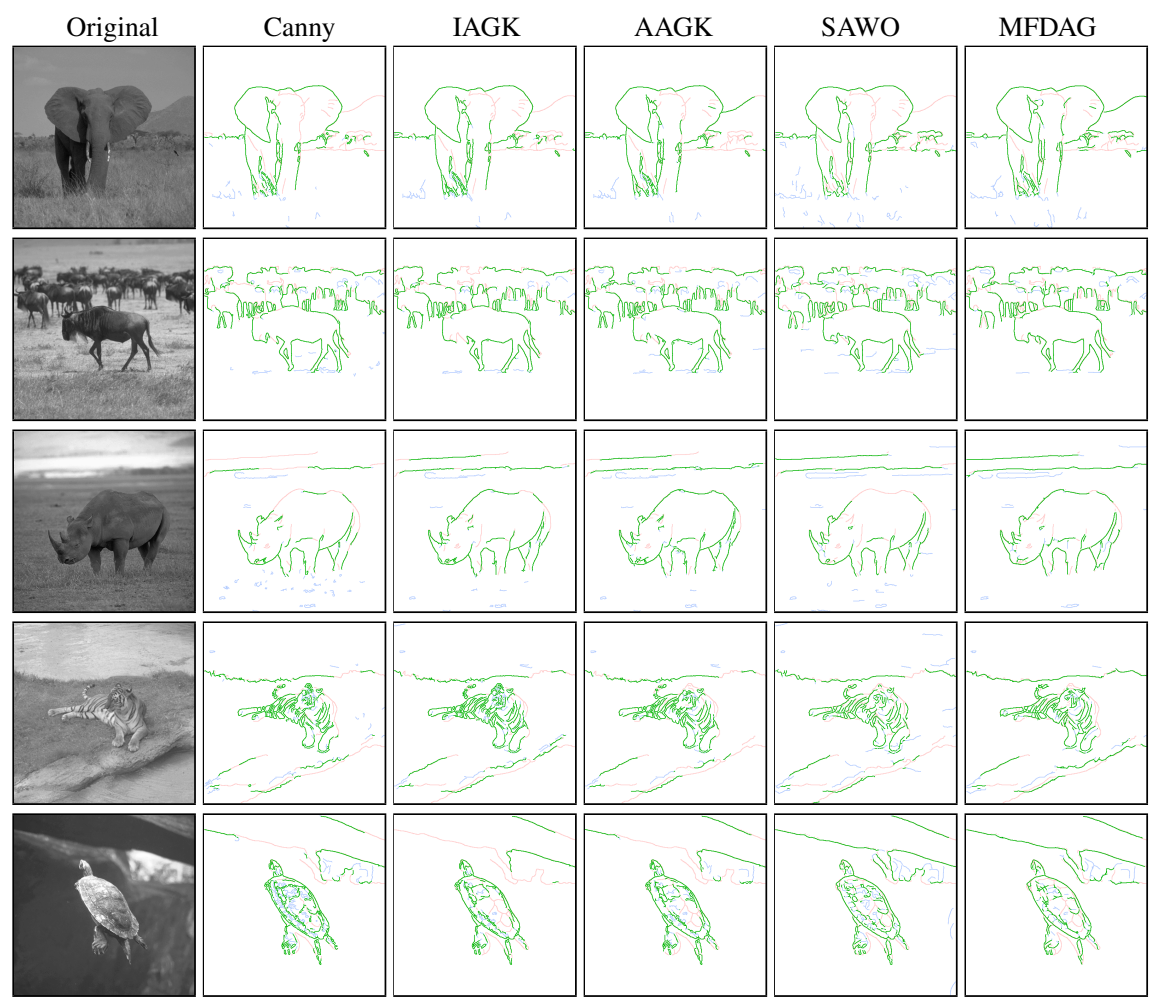

Fig. 8: Samples of detection results obtained by different methods on the RuG40 dataset. Green pixels represent true positive detection pixels, blue ones stand for false positive detection pixels and red ones denote unmatched ground truth pixels. Note that the matched ground truth pixels are also colored in green. Edges are thickened to two pixels wide for better illustration. Please zoom electronically for a better view.

\subsection{Results on the BSDS500 dataset}

We further evaluate our method on the BSDS500 dataset ${ }^{2}$, which contains 200 test images as well as their ground truth edge maps labelled by multiple annotators [28]. We illustrate several sample images and the multiple ground truth edge maps in Fig. 9. Despite the devi-

\footnotetext{
2 https://www2.eecs.berkeley.edu/Research/Projects/CS/vision/grouping/resources
} 


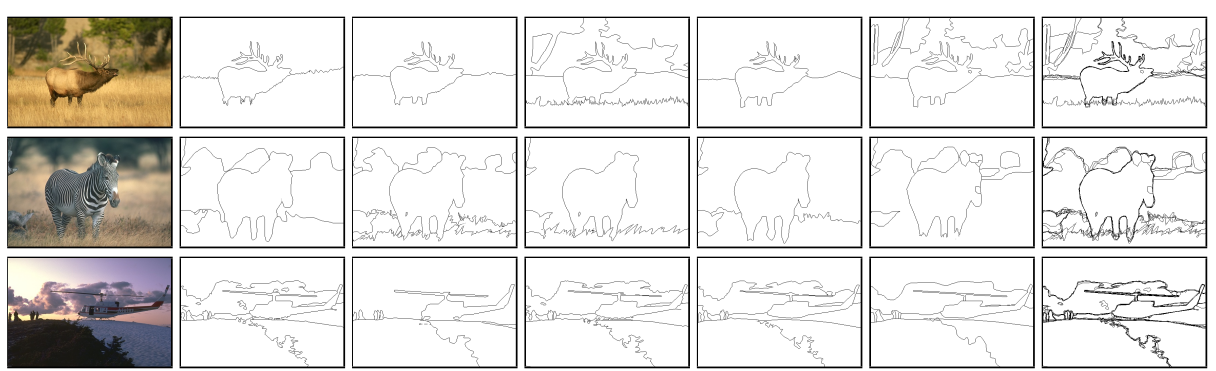

Fig. 9: Sample images and the ground truth edge maps from the BSDS500 dataset. Left column: Original images. Second to sixth columns: Ground truth edge maps labelled by different annotators for each original image. Right column: Aggregated ground truth for each original image.

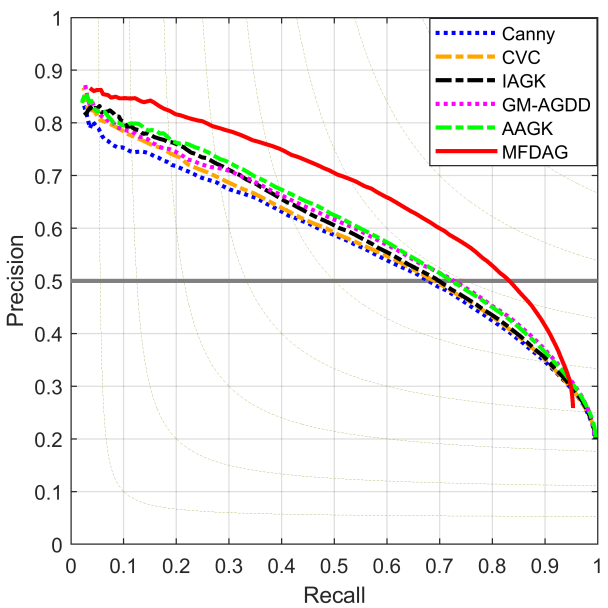

Fig. 10: PR curves of different methods obtained on the BSDS500 dataset.

ations between annotations depicted by different annotators, the overall ground truth maps reflect the edges in the original images very well. Note that there are a lot of spatially scaled edges in these natural images. In this experiment, we also compare our method with the Canny [5], CVC [14], IAGK [38], GM-AGDD [42] and AAGK [50] methods.

The obtained PR curves in Fig. 10 and the AP values in Tab. 2 demonstrate that our method achieves the largest area under the curve among all the methods. Moreover, our method also obtains larger $F_{\mathrm{ODS}}, F_{\mathrm{OIS}}$ and R50 values than the competing methods. These results are quite consistent with those obtained on the RuG40 dataset.

Samples of the detection results shown in Fig. 11 also illustrate the advantages of our method over the other ones. As can be seen, the MFDAG method detects both the thin edges and the spatially scaled edges well. For example, in the image illustrated in the first row of Fig. 11, the background shows the phenomenon of out-of-focus blur which results in some wide edges. Although not perfect, our method detects most of the edges in the background. In contrast, the competing methods miss most of the spatially scaled edges, because they have limitations in exploiting the multiscale information. 
Table 2: Results of evaluation measures obtained by different methods on the BSDS500 dataset.

\begin{tabular}{lcccc}
\hline Methods & $F_{\text {ODS }}$ & $F_{\text {OIS }}$ & AP & R50 \\
\hline Canny & 0.58 & 0.61 & 0.57 & 0.68 \\
CVC & 0.58 & 0.62 & 0.58 & 0.69 \\
IAGK & 0.58 & 0.63 & 0.59 & 0.70 \\
GM-AGDD & 0.59 & 0.63 & 0.59 & 0.73 \\
AAGK & 0.59 & 0.64 & 0.60 & 0.72 \\
MFDAG & $\mathbf{0 . 6 5}$ & $\mathbf{0 . 6 8}$ & $\mathbf{0 . 6 8}$ & $\mathbf{0 . 8 3}$ \\
\hline
\end{tabular}

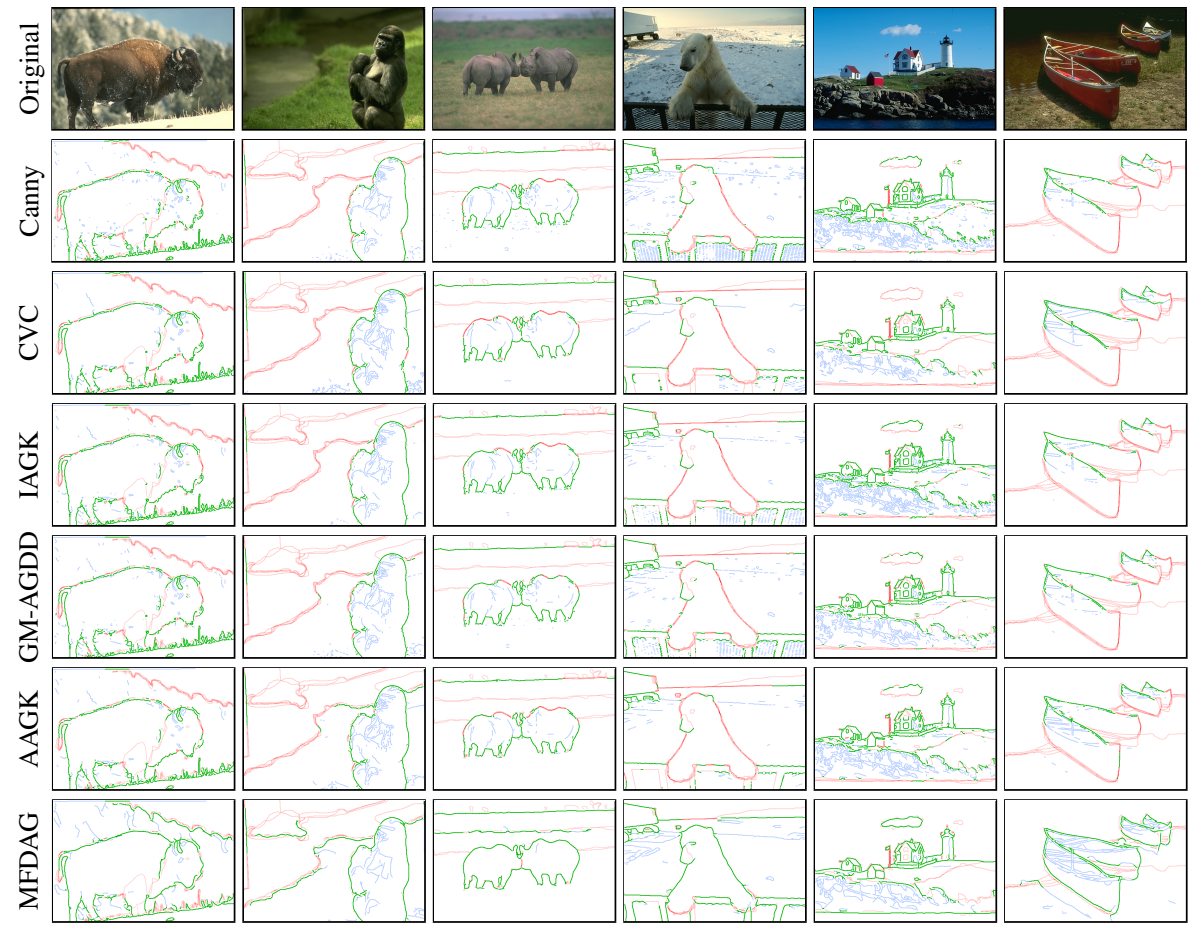

Fig. 11: Samples of detection results obtained by different methods on the BSDS500 dataset. Green pixels represent true positive detection pixels, blue ones stand for false positive detection pixels and red ones denote unmatched ground truth pixels. Note that the matched ground truth pixels are also colored in green. Edges are thickened to two pixels wide for better illustration. Please zoom electronically for a better view.

Therefore, from the results above, we conclude that the MFDAG method outperforms all the competing methods on the BSDS500 dataset. 


\section{Conclusions}

In this paper, we have presented an edge detection method based on a multiscale version of the first-order derivative of Gaussian kernel. This method is able to quantitatively measure the edge strength, the edge direction and the edge scale simultaneously while reducing the impact of noise. It is quite reliable in detecting edges with heterogeneous widths. The proposed method has been evaluated on widely used datasets. Experimental results show the benefits of the proposed method over the competing methods, including the Canny [5], CVC [14], IAGK [38], GM-AGDD [42], AAGK [50] and SAWO [24] methods.

\section{References}

1. Arbelaez, P., Maire, M., Fowlkes, C., Malik, J.: Contour detection and hierarchical image segmentation. IEEE Trans. Pattern Anal. Mach. Intell. 33(5), 898-916 (2011)

2. Atick, J.J., Redlich, A.N.: What does the retina know about natural scenes? Neural Comput. 4(2), 196210 (1992)

3. Bao, P., Zhang, L., Wu, X.: Canny edge detection enhancement by scale multiplication. IEEE Trans. Pattern Anal. Mach. Intell. 27(9), 1485-1490 (2005)

4. Basu, M.: Gaussian-based edge-detection methods-a survey. IEEE Trans. Syst. Man Cybern. Part CAppl. Rev. 32(3), 252-260 (2002)

5. Canny, J.: A computational approach to edge detection. IEEE Trans. Pattern Anal. Mach. Intell. 8(6), 679-698 (1986)

6. Coleman, S.A., Scotney, B.W., Suganthan, S.: Edge detecting for range data using Laplacian operators. IEEE Trans. Image Process. 19(11), 2814-2824 (2010)

7. Ding, L., Goshtasby, A.: On the Canny edge detector. Pattern Recognit. 34(3), 721-725 (2001)

8. Dollár, P., Zitnick, C.L.: Fast edge detection using structured forests. IEEE Trans. Pattern Anal. Mach. Intell. 37(8), 1558-1570 (2015)

9. Elder, J.H., Zucker, S.W.: Local scale control for edge detection and blur estimation. IEEE Trans. Pattern Anal. Mach. Intell. 20(7), 699-716 (1998)

10. Goldberg, A.V., Kennedy, R.: An efficient cost scaling algorithm for the assignment problem. Math. Program. 71(2), 153-177 (1995)

11. Grigorescu, C., Petkov, N., Westenberg, M.A.: Contour detection based on nonclassical receptive field inhibition. IEEE Trans. Image Process. 12(7), 729-739 (2003)

12. Guerra, C., Jurio, A., Bustince, H., Lopez-Molina, C.: Multichannel generalization of the upper-lower edge detector using ordered weighted averaging operators. J. Intell. Fuzzy Syst. 27(3), 1433-1443 (2014)

13. Jacob, M., Unser, M.: Design of steerable filters for feature detection using Canny-like criteria. IEEE Trans. Pattern Anal. Mach. Intell. 26(8), 1007-1019 (2004)

14. Koschan, A., Abidi, M.: Detection and classification of edges in color images. IEEE Signal Process. Mag. 22(1), 64-73 (2005)

15. Li, X., Chen, T.: Nonlinear diffusion with multiple edginess thresholds. Pattern Recognit. 27(8), 1029$1037(1994)$

16. Li, Y., Wang, S., Tian, Q., Ding, X.: A survey of recent advances in visual feature detection. Neurocomputing 149, 736-751 (2015)

17. Li, Z., Ahmed, E., Eltawil, A.M., Cetiner, B.A.: A beam-steering reconfigurable antenna for WLAN applications. IEEE Trans. Antennas Propag. 63(1), 24-32 (2015)

18. Lindeberg, T.: Edge detection and ridge detection with automatic scale selection. Int. J. Comput. Vis. 30(2), 117-156 (1998)

19. Lindeberg, T.: Scale selection properties of generalized scale-space interest point detectors. J. Math. Imaging Vision 46(2), 177-210 (2013)

20. Lindeberg, T.: Image matching using generalized scale-space interest points. J. Math. Imaging Vis 52(1), 3-36 (2015)

21. Liu, Y., Ming-Ming Cheng, X.H., Wang, K., Bai, X.: Richer convolutional features for edge detection. In: Proceedings of the IEEE Conference on Computer Vision and Pattern Recognition, pp. 5872-5881 (2017)

22. Lopez-Molina, C., Bustince, H., De Baets, B.: Separability criteria for the evaluation of boundary detection benchmarks. IEEE Trans. Image Process. 25(3), 1047-1055 (2016) 
23. Lopez-Molina, C., De Baets, B., Bustince, H.: Quantitative error measures for edge detection. Pattern Recognit. 46(4), 1125-1139 (2013)

24. Lopez-Molina, C., Montero, J., Bustince, H., De Baets, B.: Self-adapting weighted operators for multiscale gradient fusion. Inf. Fusion 44, 136-146 (2018)

25. Lopez-Molina, C., Vidal-Diez de Ulzurrun, G., Baetens, J.M., Van Den Bulcke, J., De Baets, B.: Unsupervised ridge detection using second order anisotropic Gaussian kernels. Signal Process. 116, 55-67 (2015)

26. Marr, D., Hildreth, E.: Theory of edge detection. In: Proceedings of Royal Society of London, vol. B207, pp. 187-217 (1980)

27. Martin, D.R.: An empirical approach to grouping and segmentation. Ph.D. thesis, University of California, Berkeley (2003)

28. Martin, D.R., Fowlkes, C.C., Malik, J.: Learning to detect natural image boundaries using local brightness, color, and texture cues. IEEE Trans. Pattern Anal. Mach. Intell. 26(5), 530-549 (2004)

29. McIlhagga, W.: The Canny edge detector revisited. Int. J. Comput. Vis. 91(3), 251-261 (2011)

30. Pan, X., Ye, Y., Wang, J., Gao, X., He, C., Wang, D., Jiang, B., Li, L.: Complex composite derivative and its application to edge detection. SIAM J. Imaging Sci. 7(4), 2807-2832 (2014)

31. Perona, P., Malik, J.: Detecting and localizing edges composed of steps, peaks and roofs. In: Proceedings of the International Conference on Computer Vision, pp. 52-57 (1990)

32. Prewitt, J.M.: Object enhancement and extraction. Picture Processing and Psychopictorics 10(1), 15-19 (1970)

33. Ray, K.: Unsupervised edge detection and noise detection from a single image. Pattern Recognit. 46(8), 2067-2077 (2013)

34. Roberts, L.G.: Machine perception of three-dimensional solids. In: Optical and Electro-Optical Information Processing, pp. 159-197. MIT Press (1965)

35. Rosenfeld, A.: A nonlinear edge detection technique 58(5), 814-816 (1970)

36. Rosenfeld, A., Thurston, M.: Edge and curve detection for visual scene analysis. IEEE Trans. Comput. 20(5), 562-569 (1971)

37. Shui, P.L., Wang, F.P.: Anti-impulse-noise edge detection via anisotropic morphological directional derivatives. IEEE Trans. Image Process. 26(10), 4962-4977 (2017)

38. Shui, P.L., Zhang, W.C.: Noise-robust edge detector combining isotropic and anisotropic Gaussian kernels. Pattern Recognit. 45(2), 806-820 (2012)

39. Shui, P.L., Zhang, W.C.: Corner detection and classification using anisotropic directional derivative representations. IEEE Trans. Image Process. 22(8), 3204-3218 (2013)

40. Sobel, I.: Camera models and machine perception. Ph.D. thesis, Stanford University (1970)

41. Torre, V., Poggio, T.A.: On edge detection. IEEE Trans. Pattern Anal. Mach. Intell. 8(2), 147-163 (1986)

42. Wang, F.P., Shui, P.L.: Noise-robust color edge detector using gradient matrix and anisotropic Gaussian directional derivative matrix. Pattern Recognit. 52, 346-357 (2016)

43. Wang, G., De Baets, B.: Edge detection based on the fusion of multiscale anisotropic edge strength measurements. In: Proceedings of the Conference of the European Society for Fuzzy Logic and Technology, vol. 3, pp. 530-536 (2017)

44. Wang, G., De Baets, B.: Superpixel segmentation based on anisotropic edge strength. Journal of Imaging 5(6) (2019)

45. Wang, G., Lopez-Molina, C., De Baets, B.: Blob reconstruction using unilateral second order Gaussian kernels with application to high-ISO long-exposure image denoising. In: Proceedings of the IEEE International Conference on Computer Vision, pp. 4817-4825 (2017)

46. Wang, G., Lopez-Molina, C., Vidal-Diez de Ulzurrun, G., De Baets, B.: Noise-robust line detection using normalized and adaptive second-order anisotropic gaussian kernels. Signal Process. 160, 252-262 (2019)

47. Xie, S., Tu, Z.: Holistically-nested edge detection. Int. J. Comput. Vis. 25, 3-18 (2017)

48. Xu, Q., Varadarajan, S., Chakrabarti, C., Karam, L.J.: A distributed Canny edge detector: Algorithm and FPGA implementation. IEEE Trans. Image Process. 23(7), 2944-2960 (2014)

49. Yang, K.F., Gao, S.B., Guo, C.F., Li, C.Y., Li, Y.J.: Boundary detection using double-opponency and spatial sparseness constraint. IEEE Trans. Image Process. 24(8), 2565-2578 (2015)

50. Zhang, W., Zhao, Y., Breckon, T.P., Chen, L.: Noise robust image edge detection based upon the automatic anisotropic Gaussian kernels. Pattern Recognit. 63, 193-205 (2017)

51. Zitnick, C.L., Dollár, P.: Edge boxes: Locating object proposals from edges. In: Proceedings of the European Conference on Computer Vision, pp. 391-405 (2014) 